\title{
A NEW METHOD FOR ASSESSMENT OF MENTAL FORAMEN RELATED LANDMARKS USING COMPUTED TOMOGRAPHY SCAN
}

\author{
Mona M. Elramady*
}

\begin{abstract}
Background: Mental foramen is the opening of mandibular canal in the mandibular body, and its place shows contra-versus. As mental foramen is an essential anatomical landmark required for implant surgery, the determination of the exact place for the mental foramen to detect exact amount of local anesthesia solution and to decrease dismissed injuries during the prosthetic surgical treatments. The determination of the situation of mental foramen according to its site in horizontal and vertical direction of mental foramen according to the adjacent premolars and molars on the skull, diagnostic radiographs, and CBCT.
\end{abstract}

Objectives: Assessment of mental foramen related landmarks using computed tomography scan by a new computerized method.

Materials and Methods: Twelve patients ranging from 45-60 years old came to the department of prosthodontics, Alexandria University, Alexandria, Egypt. The anatomical structural landmarks such as mandibular canal, mental foramen according to its site relative to the lower border of the mandible of the mandible were evaluated by radiographic CT and scores were assessed and compared at baseline, after 6 months and after 12 months. All the measurements done by Image $\mathrm{J}$ computed program which was not used in the previous studies.

Results: Anatomical landmark structures in the mandible (mandibular canal, mandibular foramen, mental foramen, and incisive canal) were detected and measurements in millimeter were recorded by using CT images.

Conclusion: An essential anatomical landmark for the dentist is the place of mental foramen so, the dentists must have attention about this anatomical landmark before any surgical procedure including mental foramen region. Computed tomography (CT) is highly essential measure to avoid any expected complication.

KEY WORD: Mental foramen, anatomical landmark, computed tomography (CT) evaluation

\footnotetext{
* Department of Prosthodontics, Pharos University, Alexandria City, Alex., Egypt
} 


\section{INTRODUCTION}

Mental foramen is the opening of mandibular canal in the mandibular body, and its place shows contra-versus. As mental foramen is an essential anatomical landmark in implant surgery, to decrease accidental problems during surgical treatments. The determination of the situation of mental foramen in horizontal and vertical dire ction of mental foramen according to the adjacent premolars and molars on the skull, diagnostic radiographs, and the exact situation of mental foramen required for enough anesthetic solution which is needed in the treatment of patients who have partially or completely lost their teeth ${ }^{(1-7)}$. So, those patients need stable anatomical landmark for detecting the foramen situation which was used by few previous studies ${ }^{(8-10)}$. The determination of mental foramen relative to the lower border of the mandible and skeleton part of the midline and its age and gender differences on CBCT projections were used on an Iranian population in a previous study ${ }^{(11)}$.

Having accurate information about anatomical structures using diagnosis of pathology needed about anatomical landmark is considered an essential diagnostic factor ${ }^{(12,13)}$.

The accidental hurt to the inferior alveolar nerve or mental nerve can lead to numbness and paresthesia of the lip and chin ${ }^{(14)}$ and other difficulties during implant surgery in the incisive canal region and symphysis when mandibular surgical procedure for implant surgery. The inferior alveolar nerve separate from the mandibular bulk at the mental foramen which forms an anterior loop after leaving a short behind pathway before get back into the mandible ${ }^{(15)}$. Actually, many surgeons work on the frontal part of the mandible (intermental area) including surgical implant placement, prosthetic surgical treatment, and screw fixation. The intermental area is a secure region for surgical operations, and the presence of incisive canal is considered a problem in this region and this occurs during the placement of implant in the anterior region. It was found that, the inferior alveolar nerve may found behind the mental foramen giving an intraosseous anterior loop. The inferior alveolar nerve and blood vessels present in the mandibular canals.
The uses of cone beam computed tomography (CBCT) and its reconstruction a virtually 3D image using a cone-shaped beam in a single scan is an essential advances in previous decayed in dental treatment ${ }^{(16-19)}$. One of the essential anatomical landmark is mental foramen (FM) present on the buccal surface of the mandible, also, in a situation superior to the mandibular canal ${ }^{(20)}$ which forms a curve during its course present anterior to the MF, also it gave sensory innervations and nutrition to the gingiva, soft tissue related to the chin and lower lip ${ }^{(21)}$.

Variable anatomical landmarks of the foramen have been introduced in previous studies. The situation of the mental foramen according to its site at the vertically is present inferior to the mandibular second premolars around its apex in the half way between the crest of the alveolar ridge and the lower border of the mandible ${ }^{(22)}$.

\section{MATERIAL AND METHODS}

Twelve patients ranging from 45-60 years old came to the department of prosthodontics, Alexandria University, Alexandria, Egypt.

The study was conducted retrospectively from CT scans of 12 patients seen in the Prosthodontics department at the College of Dentistry at Alexandria University, Alexandria, Egypt. The importance of CBCT diagnostic information before treatment is to provide the suitable implant site placement, TMJ, prosthetic surgical treatment plane. All of the scans were done for detection of situation of place of MF.

The CT examination was performed for each patient utilizing for each time with $0.5 \mathrm{~mm}$ reconstruction voxel size and the volumetric data is preserved for each patient on a computerized set linked with the $\mathrm{CT}$.

After acquisition of the volume, the DICOM data was used in order to generate a 3D volumetrically converting image for each patient using Iluma vision 3D software. According to classification done by $\mathrm{Al}$ Jasser and Nwoku (1998) ${ }^{(23)}$ it was found that the situation of the MF relative to the teeth was informed in relation to its detected situation. 
According to the radiographic examination done by oral, maxillofacial and dentist to detect the 3D volumetrically image examination; it was found that all images were examined in standard dim light condition. Both the right and left mental foramina were detected separately and recorded on a specially designed form. Descriptive statistics of various situations were used (mean and standard deviation). The results were presented according to both right and left sides and gender. The SPSS (version 21) was used for analysis. The mean and standard deviation of all the measurements were assessed. Comparison of value of all measurements was made between sides using t-test. Differences between genders were considered significant at $\mathrm{P}<0.05$.

\section{Measurement procedure}

Multiplanar reconstructions from CT were jointly assessed by two experienced researchers to detect $\mathrm{MF}$ and MF. For this purpose, DICOM files were reconstructed on computer (Samsung R522, Samsung
Electronics, South Korea) using 3D visualization and measurement software Carestream ${ }^{\circledR}$ CS 3D imaging software v3.2.12 (Carestream Health Inc., Rochester, NY, USA).

The place of MF was evaluated by $\mathrm{CT}$ images as follows between the apices of inferior teeth starting from $1^{\text {st }}$ molar to $1^{\text {st }}$ premolar. The relation of the MF and MF to the mandibular body was evaluated by measuring the distances from mental foramen right and mental foramen left (MF-MF), length at Medline, mental foramen width (MF-W), mental foramen height (MF-H), alveolar crest lower border (ALVR C. - LB), alveolar crest mental foramen lower border (ALVR C. - MF - LB), mental foramen alveolar crest (MF - ALVR C.) and mental foramen lower border $(\mathrm{MF}-\mathrm{LB})$.

Dental status was classified into two groups for right and left sides. All the patients were male partially dentate ${ }^{(24)}$. (Figure 1)

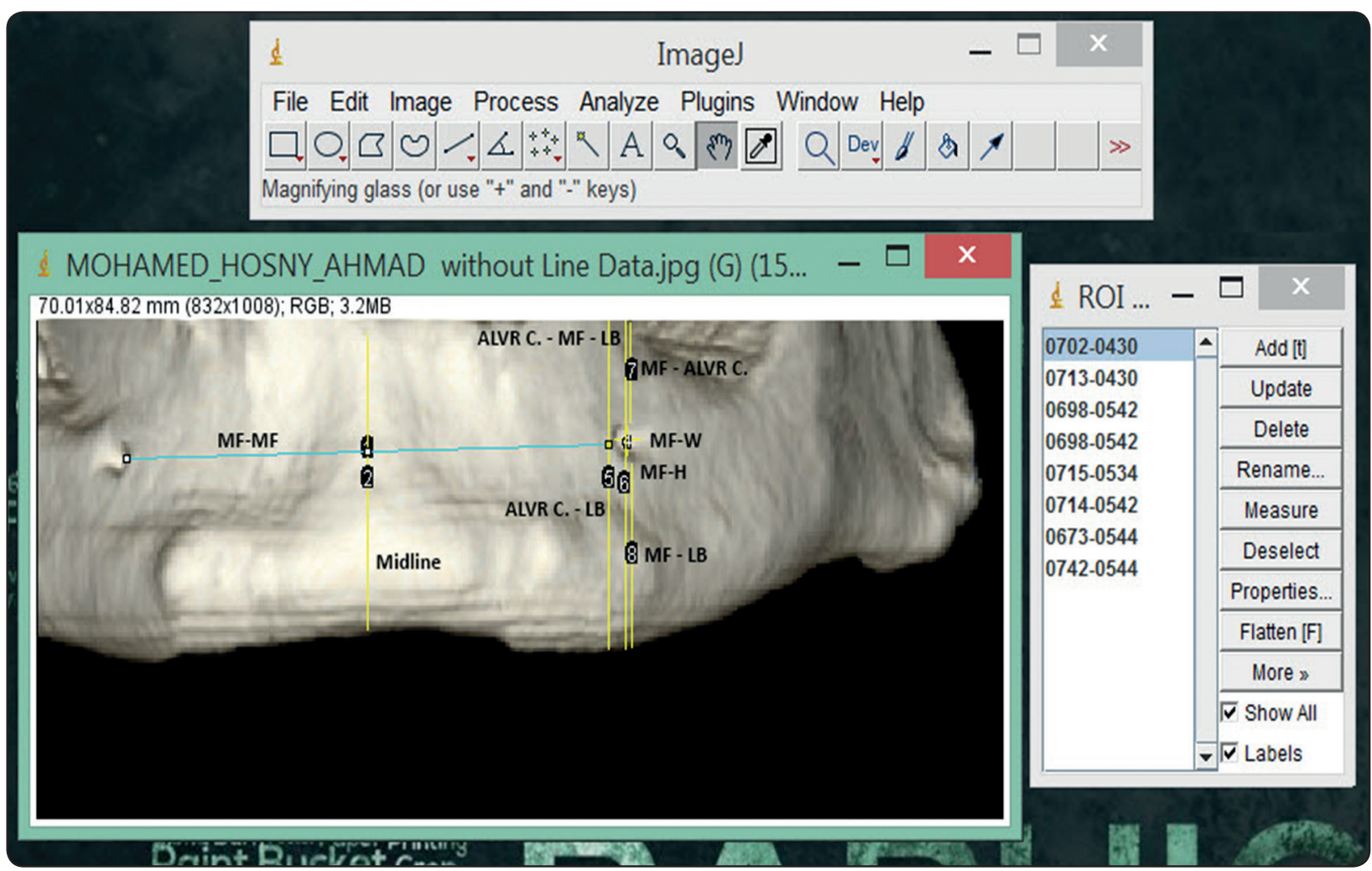

Fig. (1): Mental foramen anatomical landmark using CT scan by image J program. 


\section{RESULT}

Anatomical structures in the mandible (mandibular canal, mandibular foramen, mental foramen, and incisive canal) were detected and measurements in millimeter were recorded by using $\mathrm{CT}$ images and the measurements were compared at baseline, after 6 months and after 12 months which measured at MF-MF, Medline, MF-W, MF-H, ALVR C. - LB, ALVR C. - MF - LB, MF - ALVR C. and MF - LB.

Data were fed to the computer and analyzed using IBM SPSS software package version 20.0. (Armonk, NY: IBM Corp). The KolmogorovSmirnov was used to verify the normality of distribution of variables, Paired t-test was assessed for comparison between right and left for normally distributed quantitative variables. Significance of the obtained results was judged at the 5\% level. Table 1

The means and standard deviation for evaluation of the mental foramen $\mathrm{CT}$ at baseline at MF-MF, Medline, MF-W, MF-H, ALVR C. - LB, ALVR C. - MF - LB, MF - ALVR C. and MF - LB for the right side was $17.9 \pm 1.2,11.8 \pm 1.5,0.7 \pm 0.2,0.7$ $\pm 0.2,10.1 \pm 1.1,9.7 \pm 1.2,3.3 \pm 1.8$, and $5.4 \pm 1$ while for the left side it was resulted that $18.1 \pm 1.1$, $12 \pm 1.5,0.6 \pm 0.1,0.7 \pm 0.2,11.1 \pm 1.1,11 \pm 1.1$,
$3.6 \pm 1.5$ and $5.3 \pm 0.7$.

The means and standard deviation for the mental foramen CT After six months at MF-MF, Medline, MF-W, MF-H, ALVR C. - LB, ALVR C. - MF LB, MF - ALVR C. and MF - LB for the right side was $17.7 \pm 1.3,11.7 \pm 1.5,0.7 \pm 0.2,0.7 \pm 0.2,9.8$ $\pm 1.1,9.5 \pm 1.2,3.2 \pm 1.8$ and $5.2 \pm 0.9$ while for the left side it was resulted that $17.9 \pm 1.1,111.8 \pm 1.5$, $0.6 \pm 0.1,0.7 \pm 0.3,10.6 \pm 0.9,10.5 \pm 1,3.4 \pm 1.4$ and $5.1 \pm 0.6$.

The means and standard deviation for the mental foramen CT After 12 months at MF-MF, Medline, MF-W, MF-H, ALVR C. - LB, ALVR C. - MF LB, MF - ALVR C. and MF - LB for the right side was $17.4 \pm 1.4,11.4 \pm 1.5,0.6 \pm 0.2,0.6 \pm 0.2,9.6$ $\pm 1.1,9.2 \pm 1.2,3.5 \pm 2.1$ and $4.9 \pm 1.1$ while for the left side it was resulted that $17.6 \pm 1,11.5 \pm 1.5$, $0.6 \pm 0.1,0.7 \pm 0.2,10.4 \pm 0.9,10.2 \pm 1.1,3.2 \pm 1.4$ and $5 \pm 0.7$.

There was no statistically significance difference between right and left sides at the following sites; MF-MF, Medline, MF-W, MF-H, MF-ALVR C. and MF-LB but there was a significant different right and left sides at ALVR C.-LB at baseline ( $\mathrm{p}=0.028)$ and ALVR C.-MF-LB which was found at baseline (0.009), after 6 months (0.030) and after 12 months (0.032).

TABLE (1): Comparison between right and left according to mental foramen CT

\begin{tabular}{|c|c|c|c|c|}
\hline Mental Foramen CT & $\begin{array}{c}\text { Right } \\
(n=12)\end{array}$ & $\begin{array}{c}\text { Left } \\
(n=12) \\
\end{array}$ & $\mathbf{t}$ & $\mathbf{p}$ \\
\hline \multicolumn{5}{|l|}{ MF-MF } \\
\hline At baseline & $17.9 \pm 1.2$ & $18.1 \pm 1.1$ & 1.998 & 0.071 \\
\hline After six months & $17.7 \pm 1.3$ & $17.9 \pm 1.1$ & 1.282 & 0.226 \\
\hline After 12 months & $17.4 \pm 1.4$ & $17.6 \pm 1$ & 1.638 & 0.130 \\
\hline \multicolumn{5}{|l|}{ Medline } \\
\hline At baseline & $11.8 \pm 1.5$ & $12 \pm 1.5$ & 0.361 & 0.725 \\
\hline After six months & $11.7 \pm 1.5$ & $11.8 \pm 1.5$ & 0.389 & 0.705 \\
\hline After 12 months & $11.4 \pm 1.5$ & $11.5 \pm 1.5$ & 0.309 & 0.763 \\
\hline \multicolumn{5}{|l|}{ MF-W } \\
\hline At baseline & $0.7 \pm 0.2$ & $0.6 \pm 0.1$ & 0.443 & 0.666 \\
\hline
\end{tabular}




\begin{tabular}{|c|c|c|c|c|}
\hline After six months & $0.7 \pm 0.2$ & $0.6 \pm 0.1$ & 0.486 & 0.636 \\
\hline After 12 months & $0.6 \pm 0.2$ & $0.6 \pm 0.1$ & 0.515 & 0.616 \\
\hline \multicolumn{5}{|l|}{ MF-H } \\
\hline At baseline & $0.7 \pm 0.2$ & $0.7 \pm 0.2$ & 0.283 & 0.782 \\
\hline After six months & $0.7 \pm 0.2$ & $0.7 \pm 0.3$ & 0.612 & 0.553 \\
\hline After 12 months & $0.6 \pm 0.2$ & $0.7 \pm 0.2$ & 0.404 & 0.694 \\
\hline \multicolumn{5}{|l|}{ ALVR C. - LB } \\
\hline At baseline & $10.1 \pm 1.1$ & $11.1 \pm 1.1$ & $2.537^{*}$ & $0.028^{*}$ \\
\hline After six months & $9.8 \pm 1.1$ & $10.6 \pm 0.9$ & 2.197 & 0.050 \\
\hline After 12 months & $9.6 \pm 1.1$ & $10.4 \pm 0.9$ & 2.197 & 0.050 \\
\hline \multicolumn{5}{|l|}{ ALVR C. - MF - LB } \\
\hline At baseline & $9.7 \pm 1.2$ & $11 \pm 1.1$ & $3.135^{*}$ & $0.009^{*}$ \\
\hline After six months & $9.5 \pm 1.2$ & $10.5 \pm 1$ & $2.496^{*}$ & $0.030^{*}$ \\
\hline After 12 months & $9.2 \pm 1.2$ & $10.2 \pm 1.1$ & $2.458^{*}$ & $0.032^{*}$ \\
\hline \multicolumn{5}{|l|}{ MF - ALVR C. } \\
\hline At baseline & $3.3 \pm 1.8$ & $3.6 \pm 1.5$ & 0.710 & 0.493 \\
\hline After six months & $3.2 \pm 1.8$ & $3.4 \pm 1.4$ & 0.498 & 0.628 \\
\hline After 12 months & $3.5 \pm 2.1$ & $3.2 \pm 1.4$ & 0.564 & 0.584 \\
\hline \multicolumn{5}{|l|}{ MF - LB } \\
\hline At baseline & $5.4 \pm 1$ & $5.3 \pm 0.7$ & 0.105 & 0.918 \\
\hline After six months & $5.2 \pm 0.9$ & $5.1 \pm 0.6$ & 0.494 & 0.631 \\
\hline After 12 months & $4.9 \pm 1.1$ & $5 \pm 0.7$ & 0.204 & 0.842 \\
\hline
\end{tabular}

Data was expressed by Mean \pm SD.

p: $p$ value for comparing between Right and Left t: Paired t-test

*: Statistically significant at $p \leq 0.05$

\section{DISCUSSION}

The mandibular canal has a course that starts at the mandibular foramen and terminate at the mental foramen opening ${ }^{(25)}$. So, the accurate site of the mandibular canal and the anatomical information of this region are considered as an essential osseointegrated implant sensory-receptor site in the mandible ${ }^{(26)}$.

Insertion of dental implant during the surgical prosthetic treatment for the mandibular treatment are now a logic solution ${ }^{(25)}$, and is directly related to the perfect anatomical information and proper assessment of bone quality and quantity of this region.

More anatomic landmarks sites are provided in a scanned with fine slices than any other gives by spiral
CT generally applied for dental implant treatment and provide more accurate measurement for fixtures placement, other studies have demonstrated the uses of single-slice CT for dental implant design in both mandible and maxilla ${ }^{(27)}$. More recently estimation accuracy using multislice CT have also been reported ${ }^{(28,29)}$.

The detection of MF place is critical both in diagnostic and clinical procedures. The most common places of the MF as described within many literatures which explain its situation have been found under the apex of the first premolar or consistent with the second premolar or in between them. Anatomical sites and gender differences have been reported in the literature ${ }^{(30)}$. 
The horizontal course of inferior alveolar nerve is an essential factor for detection of the situation of the site of mental foramen on panoramic radiograph. Studies have reported that in up to $25 \%$ of cases, essential structures such as the MF cannot be exactly determined when using panoramic radiographs ${ }^{(30)}$.

Uses of panoramic radiograph provide limitation in the accurate knowledge and sites of the mental foramina due to the presence of inherent magnification and geometrical distortion ${ }^{(30)}$.

Different factor can be detected when studying CBCT like in accurate situation of the patient, rotation of the tube films around the patient's with acute tilting duration rotation, so, mental foramen obviously detected in all samples as images were acquired using VR CBCT ${ }^{(30)}$.

Al-Mahalawy et al. (2017)(31) used panoramic and CBCT images, whereas Naik et al. (2017) ${ }^{(32)}$ used dry mandible and Ngeow and Yuzawati used panoramic radiography (2003) ${ }^{(33)}$ for assessment of MF situation, it was found as a line with the second premolar (situation 4) followed by situation 3 which is the same finding also reported which were examined the situation of the MF using only VR CBCT images. In 2013, Von Arx et al ${ }^{(34)}$ conducted a study to evaluate MF situation using corrected.

In 2013, Von Arx et al ${ }^{(34)}$ assessment the MF situation using corrected CBCT images and found that it was presented between the two premolars in $56 \%$ of cases, whereas Khojastepour et al ${ }^{(7)}$ in 2015 detected MF site in Iranian population using CBCT images and found that the most famous site was below the second premolar.

Volumetric rendering (VR) which is now a useful image processing tool available in most CBCT image processing software utilized by clinician in the dental field. The objective of this study is to determine the place of the MF in a group of patients and assess the symmetry on both sides using CBCT with a volume rendering imaging processing tool. The results will be compared by using Image $\mathrm{J}$ computerized software.
It was found that the diagnosis using cone beam computed tomography (CBCT) gives enough resolution to allow perfect evaluation of osseous landmarks in the maxillofacial zone and pre-surgical discovery of OPENING ${ }^{(35)}$.

By using of immediate implant placing and immediate loading to evaluate esthetic and functional as a successful treatment for the mandibular fullarch patient, Spielau et al. ${ }^{(36)}$, concluded that the using of computer assisted implantology with threedimensional virtual implant design, guided surgery, and $\mathrm{CAD} / \mathrm{CAM}$ construction of temporary and final fabrication was not easy and required elevated level of communication between dentist, technician and patient. The use of a first tooth-supported surgical guided template after removal of the teeth and then using of second surgical guided template help the first inserted implants assess to reaching the exact situation of planned implant of the previously removed tooth situation with a guided approach.

Yoon et al., ${ }^{(37)}$ concluded that the use of CBCT scan is essential diagnostic aid that can provide useful information about anatomical structures and morphological differences at the exact needed situation for the implant. And they also found that the presence of lingual concavity should be evaluated and carefully determined in case of implant placement is indicated in the posterior first molar area region of the mandible.

In our study, significant difference were observed between each side and this may be referred to small sample size and in the future, larger sample regards to age and gender may be required to get more detected results.

\section{CONCLUSION}

Mental foramen is a major landmark so, the dentists must have attention about this anatomical landmark before any surgical procedure including mental foramen region. Computed tomography is highly essential measure to avoid any expected complication. 
As a result of high majors of neurovascular damage, the mandibular canal is considered one of the most principle anatomical landmarks in the mandible. Due to The presence of inferior alveolar nerve, artery and vein running in the mandibular canal, this structure is very critical to implant surgical procedures.

Computed tomography (CT) is considered one of the major imaging factors for surgical implant it helps the production of quickly, representation and definitive images. The CT offers excellent detection for dental implant designing more than other radiographic method. The quality of its image provide improving and advances of multislice CT scanning, allowing acquiring more slices in a less shorter period of time, due to multiple detector rows, faster table speeds and the increase the powerful chance for the speed determination. And all the measurements were detected by using a new computerized methods image J system.

Determination of the vertical and horizontal situations of mental foramen using CBCT evaluation by the information gained from the stable anatomical landmarks of the mandible like the mandibular inferior border of the mandible and the midline which were found in both of dentate and edentate patients. There is a relation between the superior border in the mental foramen and the mandibular inferior border of the mandible as regards of the gender morphology but these is no sex information about the distance from the anterior border of mental foramen to midline.

The place of MF were show contra-verses between different populations and the undetection of the exact situation of the mental foramen may lead to unexpected injury to mental region causing paresthesia or anesthesia and the using of CT affects in the exact place of the situation of the mental foramen and is very essential to be used before any surgical procedure including the intermental foramen region.

\section{REFERENCES}

1. Gada SK, Nagda SJ. Assessment of situation and bilateral symmetry of occurrence of mental foramen in dentate Asian population. J Clin Diagn Res. 2014;8:203-5.

2. Haghanifar S, Rokouei M. Radiographic evaluation of the mental foramen in a selected Iranian population. Indian J Dent Res. 2009;20:150-2.

3. al Jasser NM, Nwoku AL. Radiographic study of the mental foramen in a selected Saudi population. Dentomaxillofac Radiol. 1998;27:341-3.

4. Ngeow WC, Yuzawati Y. The place of the mental foramen in a selected Malay population. J Oral Sci. 2003;45:171-5.

5. Al-Juboori M, Al-Wakeel H, SuWen F, Yun CM. Mental foramen place and its implication in dental treatment plan. World Journal of Medicine and Medical Science Research. 2014;2:35-42.

6. Aher V, Pillai P, Ali FM, Mustafa M, Ahire M, Mudhol A, Kadri M. Anatomical situation of mental foramen: a review. Glob J Med Public Health. 2012;1:61-4.

7. Khojastepour L, Mirbeigi S, Mirhadi S, Safaee A. Place of Mental Foramen in a Selected Iranian Population: A CBCT Assessment. Iran Endod J. 2015;10:117-21.

8. Agthong S, Huanmanop T, Chentanez V. Anatomical variations of the supraorbital, infraorbital, and mental foramina related to gender and side. J Oral Maxillofac Surg. 2005;63:800-4.

9. Neiva RF, Gapski R, Wang HL. Morphometric analysis of implant-related anatomy in Caucasian skulls. J Periodontol. 2004;75:1061-7.

10. Apinhasmit W, Chompoopong S, Methathrathip D, Sansuk R, Phetphunphiphat W. Supraorbital Notch/Foramen, infraorbital foramen and mental foramen in Thais: anthropometric measurements and surgical relevance. J Med Assoc Thai 2006;89:675-82.

11. Sheikhi M, Kheir MK. CBCT assessment of mental foramen situation relative to anatomical landmarks. Int $\mathrm{J}$ Dent. 2016;2016:5821048.

12. Bhaskar SN. Radiographic interpretation for the Density. $2^{\text {nd }}$ ed. St Louis: The CV. Mosby Co.; 1975. pp 78-83.

13. Juodzbalys G, Wang H, Sabalys G. Anatomy of mandibular vitalstructures. Mandibular canal and inferior alveolarneurovascular bundle in relation with dental implantology. JOral Maxillofac Res. 2010;1:1-8. 
14. Vazquez L, Saulacic N, Belser U, Bernard JP. Efficacy ofpanoramic radiographs in the preoperative planning ofposterior mandibular implants: a prospective clinical study of 1527 consecutively treated patients. Clin Oral Implants Res. 2008;19:81-5.

15. Gerlach NL, Meijer GJ, Maal TJ, Mulder J, Rangel FA, Borstlap WA, et al. Reproducibility of 3 different tracing methods based on cone beam computed tomography in determining the anatomical situation of the mandibular canal. J Oral Maxillofac Surg. 2010;68:811-7.

16. Scarfe WC, Farman AG, Sukovic P. Clinical applications of conebeam computed tomography in dental practice. $\mathrm{J}$ Can Dent Assoc. 2006;72:75-80.

17. Scarfe WC, Farman AG. What is cone-beam CT and how does it work? Dent Clin North Am. 2008;52:707-30.

18. Miracle AC, Mukherji SK. Cone beam CT of the head and neck, part 1: physical principles. Am J Neuroradiol. 2009;30:1088-95.

19. Miracle AC, Mukherji SK. Cone beam CT of the head and neck, part 2: clinical applications. Am J Neuroradiol. 2009;30:1285-92.

20. Fishel D, Buchner A, Hershkowith A, Kaffe I. Roentgenologic study of the mental foramen. Oral Surg Oral Med Oral Pathol. 1976;41:682-6.

21. Arzouman M J, Otis L, Kipnis V, Levine D. Observations of the anterior loop of the inferior alveolar canal. Int J Oral Maxillofac Implants. 1993;8:295-300.

22. Naik MK, Sahu RK, Rath B, Rath S. Morphometric study of mental and accessory mental foramen in dry adult human mandibles in Southern Odisha population. J Dent Med Sci. 2017;16:38-43.

23. Al Jasser NM, Nwoku AL. Radiographic study of the mental foramen in a selected Saudi population. Dentomaxillofac Radiol. 1998;27:341-3.

24. Muinelo-Lorenzo J, Suárez-Quintanilla JA, FernándezAlonso A, Varela-Mallou J, Suárez-Cunqueiro MM. Anatomical characteristics and visibility of mental foramen and accessory mental foramen: Panoramic radiography vs. cone beam CT. Med Oral Patol Oral Cir Bucal. 2015;20:e707-14.

25. Butterfield KJ, Dagenais M, Clokie C. Linear tomography's clinical accuracy and validity for presurgical dental implant analysis. Oral Surg Oral Med Oral Pathol Oral Radiol Endod. 1997;84:203-9.

26. McCollough $\mathrm{CH}$, Zink FE. Performance evaluation of a multislice CT system. Med Phys. 1999;26:2223-30.

27. Liang X, Jacobs R, Lambrichts I. An assessment on spiral CT scan of the superior and inferior genial spinal foramina and canals. Surg Radiol Anat. 2006;28:98-104.

28. Cavalcanti MG, Ruprecht A, Vannier MW. 3D volume rendering using multislice $\mathrm{CT}$ for dental implants. Dentomaxillofac Radiol. 2002;31:218-23.

29. Naitoh M, Katsumata A, Nohara E, Ohsaki C, Ariji E. Measurement accuracy of reconstructed 2-D images obtained by multislice helical computed tomography. Clin Oral Implants Res. 2004;15:570-4.

30. Alfaleh WM. Place of the Mental Foramen Using Volumetrically Rendered CBCT Images. JPDA, 2020, 29.01.

31. Al-Mahalawy H, Al-Aithan H, Al-Kari B, Al-Jandan B, Shujaat $S$. Determination of the situation of mental foramen and frequency of anterior loop in Saudi population. A retrospective CBCT study. Saudi Dent J. 2017;29:29-35.

32. Naik MK, Sahu RK, Rath B, Rath S. Morphometric study of mental and accessory mental foramen in dry adult human mandibles in Southern Odisha population. J Dent Med Sci. 2017;16:38-43.

33. Ngeow WC, Yuzawati Y. The place of the mental foramen in a selected Malay population. J Oral Sci. 2003;45:171-5.

34. von Arx T, Friedli M, Sendi P, Lozanoff S, Bornstein MM. Place and dimensions of the mental foramen: a radiographic analysis by using cone-beam computed tomography. J Endod. 2013;39:1522-8.

35. Katakami K, Mishima A, Shiozaki K, Shimoda S, Hamada Y, Kobayashi K. Characteristics of accessory mental foramina observed on limited cone-beam computed tomography images. J Endod. 2008;34:1441-5.

36. Spielau T, Hauschild U, Katsoulis J. Computer-assisted, template-guided immediate implant placement and loading in the mandible: a case report. BMC Oral Health. 2019; 19:55.

37. Yoon TY, Patel M, Michaud RA, Manibo AM. Cone beam computerized tomography analysis of the posterior and anterior mandibular lingual concavity for dental implant patients. J Oral Implantol. 2017;43:12-8 